ArgOn national laboratory

ANL/MCS-TM-311

\title{
Provenance Management in Swift with Implementation Details
}

Mathematics and Computer Science Division Technical Memorandum ANL/MCS-TM-311 


\begin{abstract}
About Argonne National Laboratory
Argonne is a U.S. Department of Energy laboratory managed by UChicago Argonne, LLC under contract DE-AC02-06CH11357. The Laboratory's main facility is outside Chicago, at 9700 South Cass Avenue, Argonne, Illinois 60439. For information about Argonne and its pioneering science and technology programs, see www.anl.gov.
\end{abstract}

\title{
Availability of This Report
}

This report is available, at no cost, at http://www.osti.gov/bridge. It is also available on paper to the U.S. Department of Energy and its contractors, for a processing fee, from:

U.S. Department of Energy

Office of Scientific and Technical Information

P.O. Box 62

Oak Ridge, TN 37831-0062

phone (865) 576-8401

fax (865) 576-5728

reports@adonis.osti.gov

\begin{abstract}
Disclaimer
This report was prepared as an account of work sponsored by an agency of the United States Government. Neither the United States Government nor any agency thereof, nor UChicago Argonne, LLC, nor any of their employees or officers, makes any warranty, express or implied, or assumes any legal liability or responsibility for the accuracy, completeness, or usefulness of any information, apparatus, product, or process disclosed, or represents that its use would not infringe privately owned rights. Reference herein to any specific commercial product, process, or service by trade name, trademark, manufacturer, or otherwise, does not necessarily constitute or imply its endorsement, recommendation, or favoring by the United States Government or any agency thereof. The views and opinions of document authors expressed herein do not necessarily state or reflect those of the United States Government or any agency thereof, Argonne National Laboratory, or UChicago Argonne, LLC.
\end{abstract}


ANL/MCS-TM-311

\section{Provenance Management in Swift with Implementation Details}

by

L.M.R. Gadelha Jr., ${ }^{1,2}$ B. Clifford, ${ }^{3}$ M. Mattoso, ${ }^{1}$ M. Wilde, $, 4,5$ and I. Foster ${ }^{4,5}$

${ }^{1}$ Computer and Systems Engineering Program, Federal University of Rio de Janeiro, Brazil

${ }^{2}$ National Laboratory for Scientific Computing, Brazil

${ }^{3}$ Department of Astronomy and Astrophysics, University of Chicago, USA

${ }^{4}$ Computation Institute, University of Chicago, USA

${ }^{5}$ Mathematics and Computer Science Division, Argonne National Laboratory, USA

May 2010

This work was supported in part by CAPES Grant 5205-09-3, CNPq, and the Office of Advanced Scientific Computing Research, Office of Science, U.S. Department of Energy, under Contract DE-AC02-06CH11357. 


\section{Contents}

Abstract

1 Introduction

2 Data Model 2

3 PC3: Implementation and Queries 6

4 PC3: Evaluation $\quad 15$

5 Related Work $\quad 16$

6 Concluding Remarks $\quad 16$

$\begin{array}{lr}\text { Acknowledgments } & 17\end{array}$

$\begin{array}{lr}\text { A Database Schema } & 17\end{array}$

$\begin{array}{lr}\text { References } & 18\end{array}$ 


\title{
Provenance Management in Swift with Implementation Details
}

\author{
Luiz M. R. Gadelha Jr. ${ }^{\mathrm{a}, \mathrm{b}}$, Ben Clifford, Marta Mattoso ${ }^{\mathrm{a}}$, Michael Wilde ${ }^{\mathrm{c}, \mathrm{d}}$, Ian Foster ${ }^{\mathrm{c}, \mathrm{d}}$ \\ ${ }^{a}$ Computer and Systems Engineering Program, Federal University of Rio de Janeiro, Brazil \\ ${ }^{b}$ National Laboratory for Scientific Computing, Brazil \\ ${ }^{c}$ Computation Institute, University of Chicago, USA \\ ${ }^{d}$ Mathematics and Computer Science Division, Argonne National Laboratory, USA
}

\begin{abstract}
The Swift parallel scripting language allows for the specification, execution and analysis of large-scale computations in parallel and distributed environments. It incorporates a data model for recording and querying provenance information. In this article we describe these capabilities and evaluate interoperability with other systems through the use of the Open Provenance Model. We describe Swift's provenance data model and compare it to the Open Provenance Model. We also describe and evaluate activities performed within the Third Provenance Challenge, which consisted of implementing a specific scientific workflow, capturing and recording provenance information of its execution, performing provenance queries, and exchanging provenance information with other systems. Finally, we propose improvements to both the Open Provenance Model and Swift's provenance system.
\end{abstract}

Key words: provenance, parallel scripting languages, scientific workflows

\section{Introduction}

The automation of large scale computational scientific experiments can be accomplished through the use of workflow management systems [9], parallel scripting tools [23], and related systems that allow the definition of the activities, input and output data, and data dependencies of such experiments. The manual analysis of the data resulting from their execution is usually not feasible, due to the large amount of information commonly generated by these experiments. Provenance systems can be used to facilitate this task, since they gather details about the design [14] and execution of these experiments, such as data artifacts consumed and produced by their activities. They also make it easier to reproduce an experiment for the purpose of verification.

The Open Provenance Model (OPM) [18] is an ongoing effort to standardize the representation of provenance information. It defines the entities artifact, process, and agent and the relationships used (between an artifact and a process), wasGeneratedBy (between a process and an artifact), wasControlledBy (between an agent and a process), wasTriggeredBy (between two processes), and wasDerivedFrom (between two artifacts). These relationships are used to assert causal dependencies between the entities defined in the model. A set of these assertions can be used to build a provenance graph. One of the main objectives of OPM is to allow the exchange of provenance information between systems. It also describes valid inferences that can be made from provenance graphs. More complex relationships between processes and artifacts can be derived using, for instance, transitivity.

The Swift parallel scripting system [25] [23] is a successor of the Virtual Data System (VDS) [13] [26] [6]. It allows the specification, management and execution of large-scale scientific workflows on parallel and distributed environments. The SwiftScript language is used for high-level specification of computations, it

Email addresses: lgadelha@lncc.br (Luiz M. R. Gadelha Jr.), benc@hawaga.org.uk (Ben Clifford), marta@cos.ufrj.br (Marta Mattoso), wilde@mcs.anl.gov (Michael Wilde), foster@mcs.anl.gov (Ian Foster) 
Table 1: Database relation processes.

\begin{tabular}{|l|l|}
\hline Attribute & Definition \\
\hline id & the URI identifying the process \\
\hline type & $\begin{array}{l}\text { the type of the process: execution, } \\
\text { compound procedure, function, op- } \\
\text { erator }\end{array}$ \\
\hline
\end{tabular}

has features such as data types, data mappers, dataset iteration, conditional branching, and procedural composition. It allows the manipulation of datasets in terms of their logical organization. The XML Dataset Typing and Mapping (XDTM) [19] notation is used to define mappers between this logical organization and the actual physical structure of the dataset. Procedures perform logical operations on input data, without modifying them. SwiftScript also allows procedures to be composed to define more complex computations. By analyzing the inputs and outputs of these procedures, the system determines data dependencies between them. This information is used to execute procedures that have no mutual data dependencies in parallel. Swift supports common execution managers for clustered systems and grid environments, such as Condor [11], GRAM [7], and PBS [16]. It also supports Falkon [21], an execution engine that provides high job execution throughput; and SSH [24], for executing jobs via secure remote logins. Swift logs a variety of information about each computation. This information can be exported using tools included in Swift to a relational database that uses a data model similar to OPM. Our provenance approach focuses on gathering information about the relationship between data and processes at the SwiftScript level. We do not gather information about lower level processes involved in executing a parallel script with Swift, such as moving data to computational resources, and submitting tasks to execution managers, although this is logged and could be integrated.

The objective of this paper is to present and evaluate the local and remote provenance recording and analysis capabilities of Swift, and compare them with those of other provenance systems. In the sections that follow, we describe the provenance capabilities of the Swift system and evaluate its interoperability with other systems through the use of OPM. We describe the provenance data model of the Swift system and compare it to OPM. We also describe and evaluate activities performed within the Third Provenance Challenge (PC3) which consisted of implementing and executing a scientific workflow (Pan-STARRS' [12] LoadWorkflow), gathering and recording provenance information of its execution, performing provenance queries, and exchanging provenance information with other systems.

\section{Data Model}

In Swift, data is represented by strongly-typed single-assignment variables. Data types can be atomic or composite. Atomic types are given by primitive types, such as integers or strings, or mapped types. Mapped types are used for representing and accessing data stored in local or remote files. Composite types are given by structures and arrays. In the Swift runtime, data is represented by a dataset handle. It may have as attributes a value, a filename, a child dataset handle (when it is a structure or an array), or a parent dataset handle (when it is contained in a structure or an array).

Swift processes are given by invocations of external programs, invocations of internal procedures, built-in functions, and operators. Dataset handles are produced and consumed by Swift processes.

In the Swift provenance model, dataset handles and processes are recorded, as are the relations between them (either a process consuming a dataset handle as input, or a process producing a dataset handle as output). Each dataset handle and process is uniquely identified in time and space by a URI. This information is stored persistently in a relational database. The two key relational tables used to store the structure of the provenance graph are processes, which stores brief information about processes (see table 1), and dataset_usage, which stores produced and consumed relationships between processes and dataset handles (see table 2). Other tables (see figure 1) are used to record details about each process and dataset, and other relationships such as dataset containment. 
Table 2: Database relation dataset_usage.

\begin{tabular}{|l|l|}
\hline Attribute & Definition \\
\hline process_id & $\begin{array}{l}\text { a URI identifying the process end } \\
\text { of the relationship }\end{array}$ \\
\hline dataset_id & $\begin{array}{l}\text { a URI identifying the dataset } \\
\text { handle end of the relationship }\end{array}$ \\
\hline direction & $\begin{array}{l}\text { whether the process is consuming } \\
\text { or producing the dataset handle }\end{array}$ \\
\hline param_name & $\begin{array}{l}\text { the parameter name of this rela- } \\
\text { tion }\end{array}$ \\
\hline
\end{tabular}

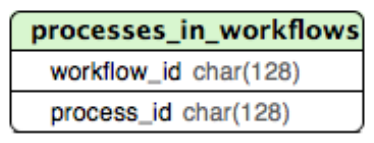

\begin{tabular}{|l|}
\hline extrainfo \\
\hline execute2id $\operatorname{char}(128)$ \\
\hline extrainfo char(1024) \\
\hline
\end{tabular}

workflow_events

workflow_id char(128)

starttime numeric

duration numeric

\begin{tabular}{|l|}
\hline executes \\
\hline id char(128) \\
\hline starttime numeric \\
\hline duration numeric \\
\hline finalstate char(128) \\
\hline app char(128) \\
\hline scratch char(128) \\
\hline
\end{tabular}

\begin{tabular}{|l|}
\hline dataset_usage \\
\hline process_id char(128) \\
\hline direction char(1) \\
\hline dataset_id char(128) \\
\hline
\end{tabular}

param_name char(128)

\begin{tabular}{|l|}
\hline known_workflows \\
\hline workflow_id char(128) \\
\hline workflow_log_filename char(128) \\
\hline version char(128) \\
\hline importstatus char(128) \\
\hline
\end{tabular}

\begin{tabular}{|l|}
\hline execute2s \\
\hline id char(128) \\
\hline execute_id char(128) \\
\hline starttime numeric \\
\hline duration numeric \\
\hline finalstate char(128) \\
\hline site char(128) \\
\hline
\end{tabular}

dataset_filenames dataset_id char(128) filename char(128) dataset_values dataset_id char(128) value char(128)

Figure 1: Swift's provenance database relations. 


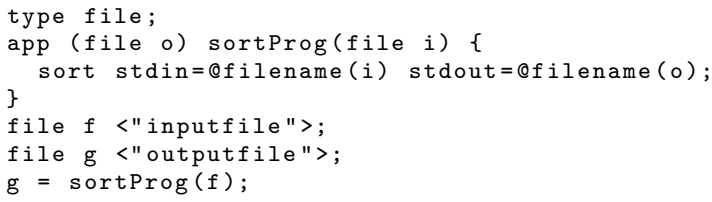

Consider the SwiftScript program in listing 1, which first describes a procedure (sortProg, which calls the external executable sort); then declares references to two files, ( $f$, a reference to inputfile, and $g$, a reference to outputfile); and finally calls the procedure sortProg. When this program is run, provenance records are generated as follows:

- a process record is generated for the initial call to the $\operatorname{sortProg}(f)$ procedure;

- a process record is generated for the @filename(i) function invocation inside sortProg, representing the evaluation of the @filename function that Swift uses to determine the physical filename corresponding to the reference $f$;

- and a process record is generated for the @filename(o) function invocation inside sortProg, again representing the evaluation of the @filename function, this time for the reference $g$.

Dataset handles are recorded for:

- the string "inputfile";

- the string "outputfile";

- the file variable $\mathrm{f}$;

- the file variable $g$;

- the filename of $i$;

- and the filename of $\mathrm{o}$.

Input and output relations are recorded as:

- the $\operatorname{sortProg}(f)$ procedure takes $f$ as an input;

- the sortProg(f) procedure produces g as an output;

- the @filename(i) function takes $f$ as an input;

- the @filename(i) function produces the filename of $f$ as an output;

- the @filename(o) function takes g as an input;

- and the @filename(o) function produces the filename of $g$ as an output.

The Swift provenance model is close to OPM, but there are some differences. Dataset handles correspond closely with OPM artifacts as immutable representations of data. However they do not correspond exactly, dataset handles do not record provenance due to aliasing, such as when accessing arrays. Section 4 discusses this issue in more detail. The OPM entity "agent" is currently not represented in Swift's provenance model, however this could be represented, for instance, by the identity of the user that runs a workflow.

Except for wasControlledBy, the dependency relationships defined in OPM can be derived from the dataset_usage database relation. It explicitly stores the used and wasGeneratedBy relationships. Table 3 


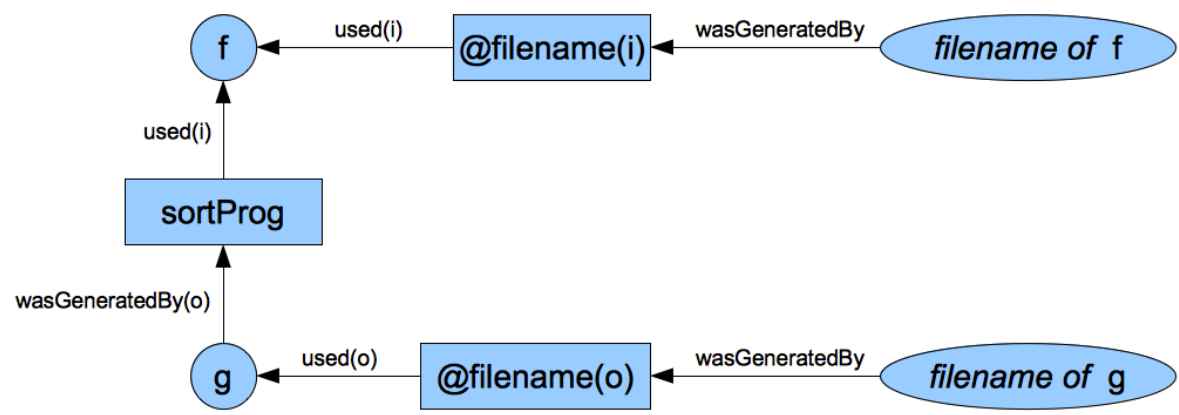

Figure 2: Provenance relationships of a sortProg execution.

Table 3: Equivalence between tuples in the dataset_usage table and OPM relationships.

\begin{tabular}{|c|c|c|c|c|}
\hline \multicolumn{4}{|c|}{ Tuple in the dataset_usage table } & \multirow{2}{*}{ OPM equivalent } \\
\hline process_id & dataset_id & direction & param_name & \\
\hline $\operatorname{sortProg}(f)$ & $f$ & In & $i$ & used(i) \\
\hline $\operatorname{sortProg}(f)$ & $\mathrm{g}$ & Out & 0 & sortProg wasGeneratedBy(0) \\
\hline Qfilename (i) & $f$ & In & $i$ & @filename(i) \\
\hline Qfilename (i) & filename of $f$ & Out & result & @filename(i) $\triangleleft$ wasGeneratedBy \\
\hline Qfilename (o) & $\mathrm{g}$ & In & o & @filename(o) \\
\hline Qfilename (i) & filename of $g$ & Out & result & @filename(o) wasGeneratedBy \\
\hline
\end{tabular}

shows the equivalence between tuples stored in the dataset_usage table and OPM relationships. wasTriggeredBy and wasDerivedFrom dependency relationships can also be inferred from database_usage, in the sortProg example we have, for instance, $f \stackrel{\text { wasDerivedFrom }}{\longleftarrow} g$. Figure 2 shows the provenance relationships captured by Swift's provenance system for the sortProg example using OPM notation.

One of the main concerns with using a relational model for representing provenance is the need for querying over the transitive relation expressed in the dataset_usage table. For example, after executing the SwiftScript code in listing 2, it might be desirable to find all dataset handles that lead to c: that is, a and b. However simple SQL queries over the dataset_usage relation can only go back one step, leading to the answer $\mathrm{b}$ but not to the answer $\mathrm{a}$. To address this problem, we generate a transitive closure table by an incremental evaluation system [10]. This approach makes it straightforward to query over transitive relations using natural SQL syntax, at the expense of larger database size and longer import time.

Swift's provenance data model is not dependent on a particular database model. A number of other forms were briefly experimented with during development [5]. The two most developed and interesting models were XML and Prolog. XML provides a semi-structured tree form for data. A benefit of this approach is that new data can be added to the database without needing an explicit schema to be known to the database. In addition, when used with a query language such as XPath, certain transitive queries become straightforward with the use of the // operator of XPath. Representing the data as Prolog tuples is a different representation than a traditional database, but provides a query interface that can express interesting queries flexibly. 


\section{PC3: Implementation and Queries}

One of the main goals of PC3 was to evaluate OPM as a mechanism for interoperability between provenance systems. An astronomy workflow from the Pan-STARRS [12] project, called LoadWorkflow, was used for this purpose. It receives a set of CSV files containing astronomical data, stores the contents of these files in a relational database, and performs a series of validation steps. This workflow makes extensive use of conditional and loop flow controls and database operations. Database operations are somewhat outside the scope of usual Swift applications, which are generally file-oriented. A Java implementation of the component applications of LoadWorkflow was provided in the Provenance Challenge Wiki [2]. These components are declared in the SwiftScript implementation of the workflow as external application procedures. The procedural body of the SwiftScript code closely follows the LoadWorkflow specification since Swift has native support for decision and loop controls, given by the if and foreach constructs.

Initially, the mapped types used in the workflow are declared. Mapped types refer to data objects that do not reside in the main memory, which is the case for data files. Most of the inputs and outputs of the Java implementation of the workflow activities are files in XML format, represented in our Swift implementation as xmlfile. In order to manipulate the input and output values we had to convert some of these files into plain text files, represented as textfile, and then read them using the readData SwiftScript function.

type xmlfile;

type textfile;

The following part consists of using app declarations to define the workflow's component applications. This allows the invocation of executable applications of the Java implementation of the workflow from Swift. They define the applications' inputs and outputs, which are XML files in the LoadWorkflow case, and provide a reference that will allow Swift to find the actual application executable by looking at its application catalog. These app declarations are given by ps_load_executable, ps_load_executable_threaded, ps_load_executable_db, and compact_database. ps_load_executable_db and compact_database also have a reference to the LoadWorkflow database as input, which is given also by an XML file. The subsequent declarations are used to manipulate an XML file in order to extract boolean values, count entries, and extract entries. Finally, the stop app declaration simply refer to a shell script that returns an error code and is used to halt the workflow execution.

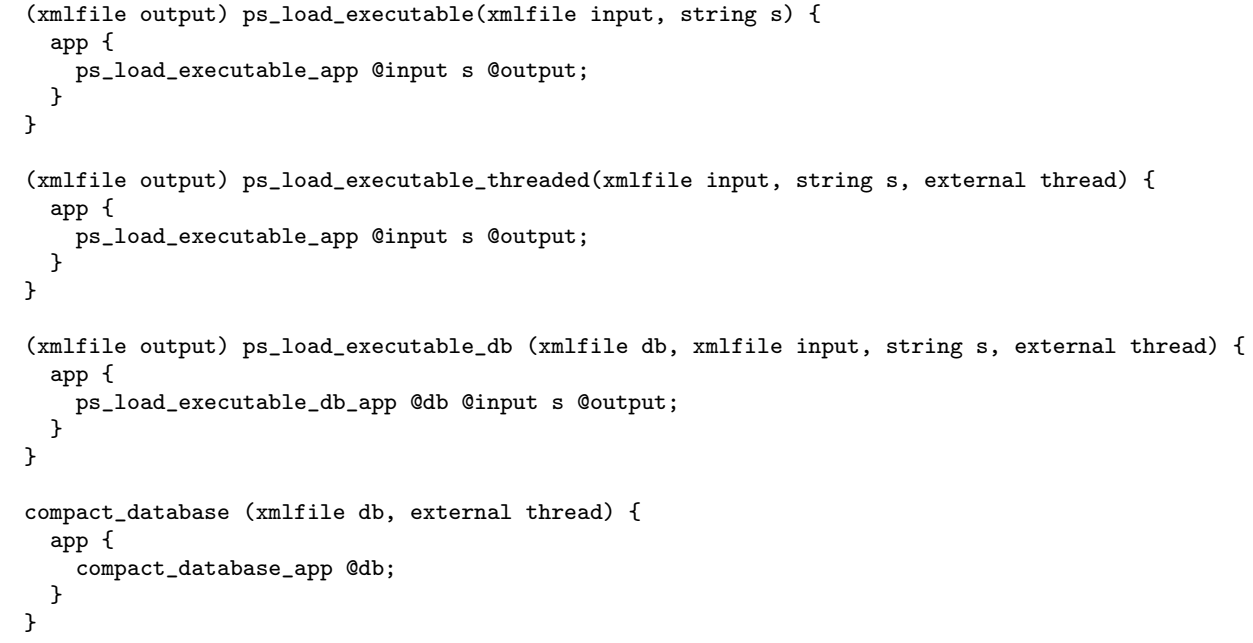


Table 4: LoadWorkflow Activities - Pre-Load Section

\begin{tabular}{|c|c|c|}
\hline Activity & Input & Output \\
\hline $\begin{array}{l}\text { IsCSVReadyFileExists: Verifies } \\
\text { if the CSV root directory and the } \\
\text { csv_ready.csv file exist. }\end{array}$ & $\begin{array}{l}\text { string CSVRootPathInput, con- } \\
\text { taining the path to the CSV root } \\
\text { directory. }\end{array}$ & $\begin{array}{l}\text { boolean IsCSVReadyFileExist- } \\
\text { sOutput, which is true if the ver- } \\
\text { ification succeeds, or false other- } \\
\text { wise. }\end{array}$ \\
\hline $\begin{array}{l}\text { ReadCSVReadyFile: For each } \\
\text { file listed in cSv_ready.csv, it } \\
\text { creates a CSVFileEntry, which } \\
\text { consists of the path to the CSV } \\
\text { file to be loaded, the path to the } \\
\text { CSV header file containing the } \\
\text { list of data columns, the num- } \\
\text { ber of rows in the file, the tar- } \\
\text { get database table, and the MD5 } \\
\text { hash function value of the file. } \\
\text { The columns names field is not } \\
\text { populated by this activity. }\end{array}$ & $\begin{array}{l}\text { string CSVRootPathInput, con- } \\
\text { taining the path to the CSV root } \\
\text { directory. }\end{array}$ & $\begin{array}{l}\text { list ReadCSVReadyFileOutput } \\
\text { of CSVFileEntry elements read } \\
\text { from csv_ready.csv. }\end{array}$ \\
\hline $\begin{array}{l}\text { IsMatchCSVFileTable: Verifies } \\
\text { if the tables to be loaded have } \\
\text { corresponding data files. }\end{array}$ & $\begin{array}{l}\text { list FileEntriesInput of CSV- } \\
\text { FileEntry elements read from } \\
\text { csv_ready.csv. }\end{array}$ & $\begin{array}{l}\text { boolean IsMatchCSVFileTable- } \\
\text { sOutput, which is true if the } \\
\text { tables have corresponding CSV } \\
\text { files, or false otherwise. }\end{array}$ \\
\hline $\begin{array}{l}\text { IsExistsCSVFileTable: Verifies if } \\
\text { CSV data file and CSV header } \\
\text { exist. }\end{array}$ & CSVFileEntry FileEntryInput. & $\begin{array}{l}\text { boolean IsExistsCSVFileOutput, } \\
\text { which is true if the CSV data } \\
\text { file and CSV header files exist, } \\
\text { or false otherwise. }\end{array}$ \\
\hline $\begin{array}{l}\text { ReadCSVFileColumnNames: } \\
\text { Reads the list of column names } \\
\text { present in the CSV data file } \\
\text { from the CSV header file. }\end{array}$ & CSVFileEntry FileEntryInput. & $\begin{array}{l}\text { CSVFileEntry FileEntryOutput, } \\
\text { which results from updating the } \\
\text { columns names field in the in- } \\
\text { put using the values listed in the } \\
\text { CSV header file. }\end{array}$ \\
\hline $\begin{array}{l}\text { IsMatchCSVFileColumnNames: } \\
\text { Verifies if the columns expected } \\
\text { for a target table are present in } \\
\text { the CSV data file. }\end{array}$ & $\begin{array}{l}\text { CSVFileEntry FileEntryInput, } \\
\text { with the columns names field } \\
\text { populated. }\end{array}$ & $\begin{array}{l}\text { boolean IsMatchCSV- } \\
\text { FileColumnNamesOutput, } \\
\text { which is true if column names } \\
\text { listed in the CSV data files } \\
\text { match the column names for the } \\
\text { target database table, or false } \\
\text { otherwise. }\end{array}$ \\
\hline
\end{tabular}


Table 5: LoadWorkflow Activities - Load Section

\begin{tabular}{|l|l|l|}
\hline Activity & Input & Output \\
\hline $\begin{array}{l}\text { CreateEmptyLoadDB: Creates } \\
\text { the database to which the CSV } \\
\text { data files will be loaded. It re- } \\
\text { turns a DatabaseEntry, which is } \\
\text { a reference to the database con- } \\
\text { taining its name and connection } \\
\text { information. }\end{array}$ & $\begin{array}{l}\text { string JobID, a unique job iden- } \\
\text { tifier for the batch of CSV data }\end{array}$ & $\begin{array}{l}\text { A DatabaseEntry CreateEmpty- } \\
\text { LoadDBOutput. }\end{array}$ \\
\hline $\begin{array}{l}\text { LoadCSVFileIntoTable: Loads a } \\
\text { CSV data file into the corre- } \\
\text { sponding database table. }\end{array}$ & $\begin{array}{l}\text { DatabaseEntry DBEntry, con- } \\
\text { taining target table to load the } \\
\text { CSV data file into. CSVFileEn- } \\
\text { try FileEntry, refering to the } \\
\text { CSV data file to be loaded. }\end{array}$ & $\begin{array}{l}\text { Output, which is true is the load } \\
\text { was successful, or false otherwise. }\end{array}$ \\
\hline $\begin{array}{l}\text { UpdateComputedColumns: Up- } \\
\text { dates the computed columns in } \\
\text { the table that was loaded. These } \\
\text { columns are indicated by the } \\
\text { value -999 in the CSV data file. }\end{array}$ & $\begin{array}{l}\text { DatabaseEntry DBEntry, with } \\
\text { the target table already loaded } \\
\text { from the CSV data file. CSV- } \\
\text { FileEntry FileEntry, containing } \\
\text { the name of target table in the } \\
\text { database to update. }\end{array}$ & $\begin{array}{l}\text { boolean } \\
\text { if the columns }\end{array}$ \\
updated, or false otherwise. \\
\hline
\end{tabular}

Table 6: LoadWorkflow Activities - Post-Load Section

\begin{tabular}{|c|c|c|}
\hline Activity & Input & Output \\
\hline $\begin{array}{l}\text { IsMatchTableRowCount: } \\
\text { Checks if number of rows } \\
\text { loaded into table matches the } \\
\text { expected. }\end{array}$ & $\begin{array}{l}\text { DatabaseEntry DBEntry, where } \\
\text { the target table is loaded and up- } \\
\text { dated. CSVFileEntry FileEntry, } \\
\text { containing the expected number } \\
\text { of rows in the CSV data file and } \\
\text { the target database table name. }\end{array}$ & $\begin{array}{l}\text { bool IsMatchTableRow- } \\
\text { CountOutput, which is true } \\
\text { if the number of rows in the tar- } \\
\text { get table matches the expected } \\
\text { number of rows in the CSV data } \\
\text { file. }\end{array}$ \\
\hline $\begin{array}{l}\text { IsMatchTableColumnRanges: } \\
\text { Checks if the data loaded into } \\
\text { database table columns is within } \\
\text { the range of values expected. }\end{array}$ & $\begin{array}{l}\text { DatabaseEntry DBEntry, where } \\
\text { the target table is loaded and up- } \\
\text { dated. CSVFileEntry FileEntry, } \\
\text { containing the name of target ta- } \\
\text { ble in the database to validate } \\
\text { columns ranges. }\end{array}$ & $\begin{array}{l}\text { bool IsMatchTableColumn- } \\
\text { RangesOutput, which is true if } \\
\text { the data values of the columns } \\
\text { in the target table are within } \\
\text { the expected range, or false } \\
\text { otherwise. }\end{array}$ \\
\hline $\begin{array}{l}\text { CompactDatabase: Compacts } \\
\text { the database before concluding } \\
\text { the workflow. }\end{array}$ & $\begin{array}{l}\text { DatabaseEntry DBEntry, where } \\
\text { all tables are loaded and vali- } \\
\text { dated. }\end{array}$ & None. \\
\hline
\end{tabular}




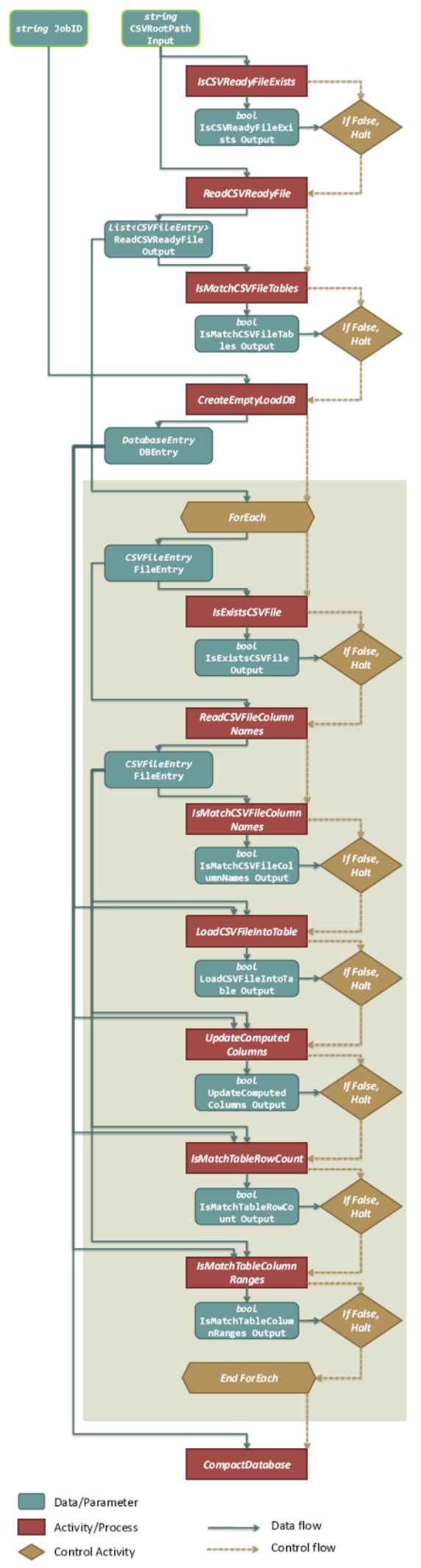

Figure 3: LoadWorkflow. Source: PC3 web site [1]. 


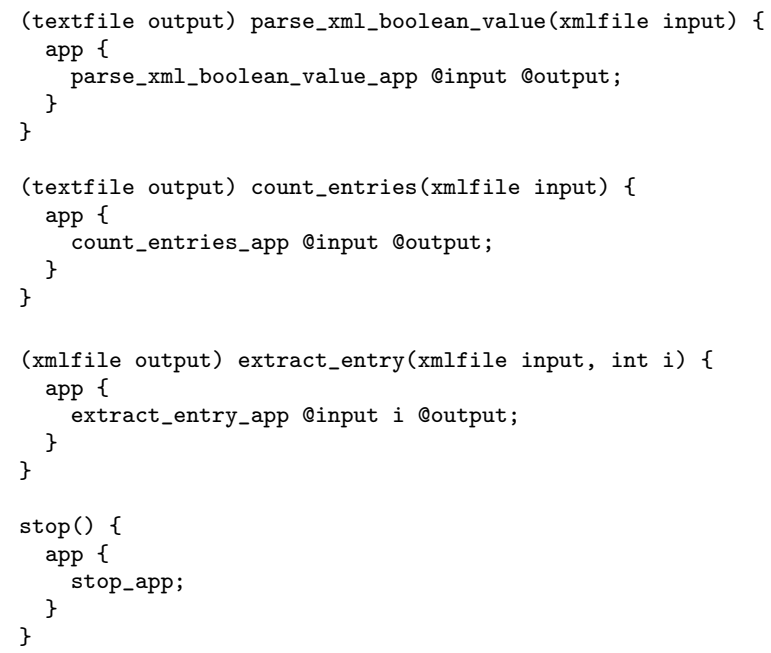

The next part of the SwiftScript code is used for the declaration of compound procedures, which invoke other SwiftScript procedures instead of component programs. The extract_boolean procedure reads a text file and extracts the boolean value it contains. The checkvalid procedure simply tests a boolean value and halts the workflow if it is false. ps_load_executable_boolean and ps_load_executable_db_boolean are used to execute a workflow activity, they return a boolean value as output. The remaining procedures implement actual workflow activities by calling the other SwiftScript procedures defined.

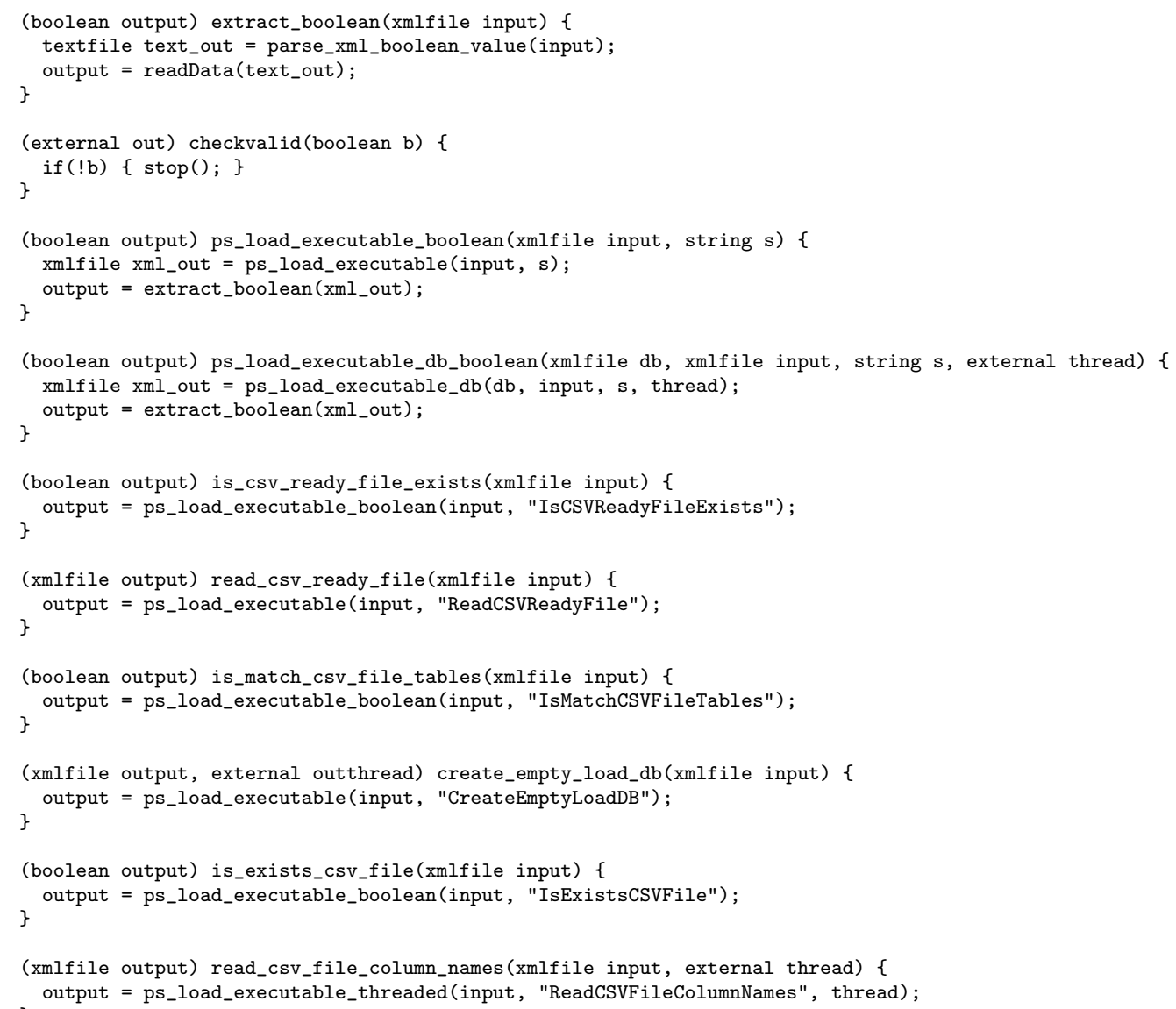




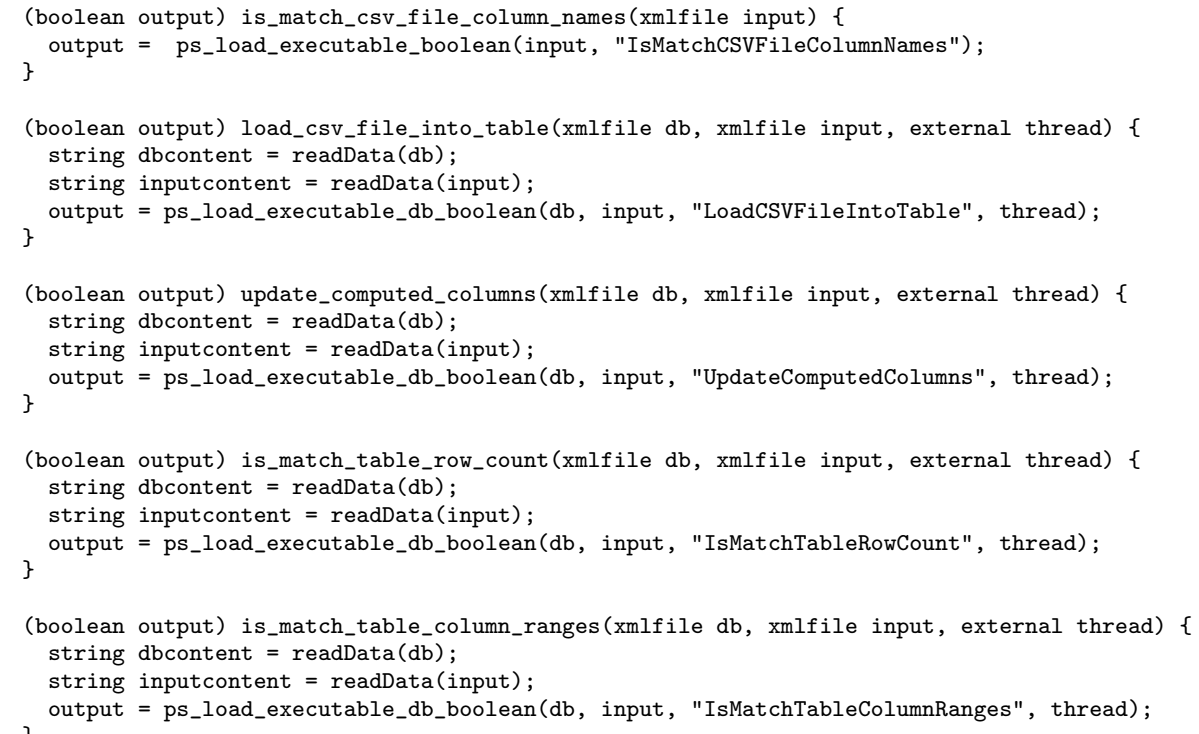

The subsequent piece of Swiftscript code is used for variable declarations. The workflow receives two files as input arguments, one containing the path to the CSV root directory and another one containing a job identifier. These values are received by the csv_root_path_input_arg and job_id_arg variables. The csv_root_path_input and job_id mapped type variables are declared and their values are declared to be contained in the files given as input arguments. The remaining variables declared in this piece of code are used to hold outputs of workflow procedures.

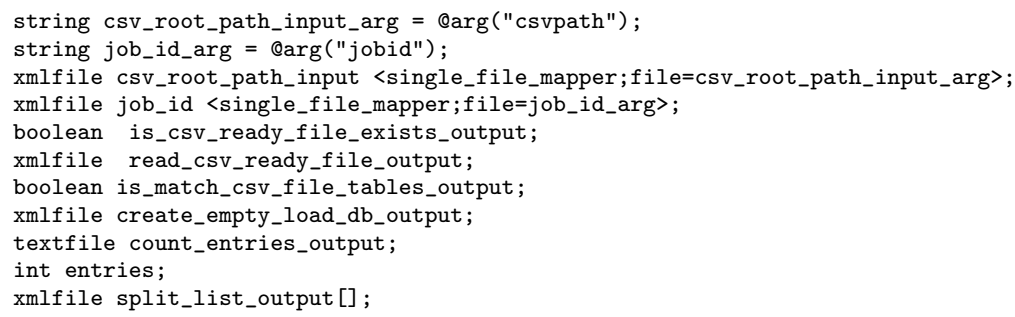

The final part of the SwiftScript code is the actual procedural portion of the LoadWorkflow implementation in Swift. It closely follows the LoadWorkflow logic since Swift has native support for decision and loop controls. The split_list_output array holds the CSV file entries that will be processed in the workflow, they are extracted from the XML file generated by the read_csv_ready_file procedure.

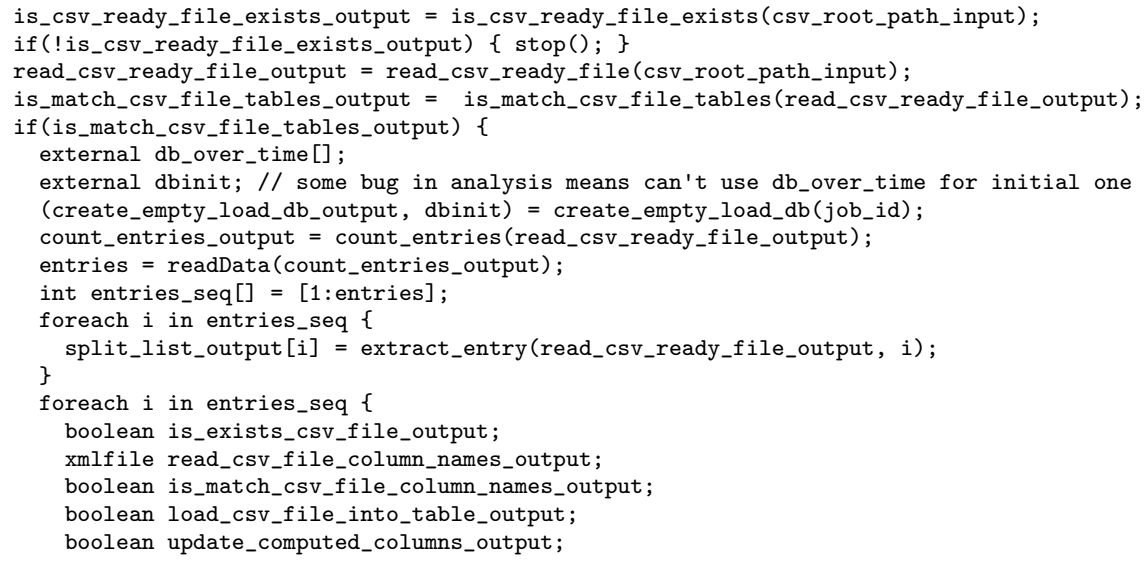




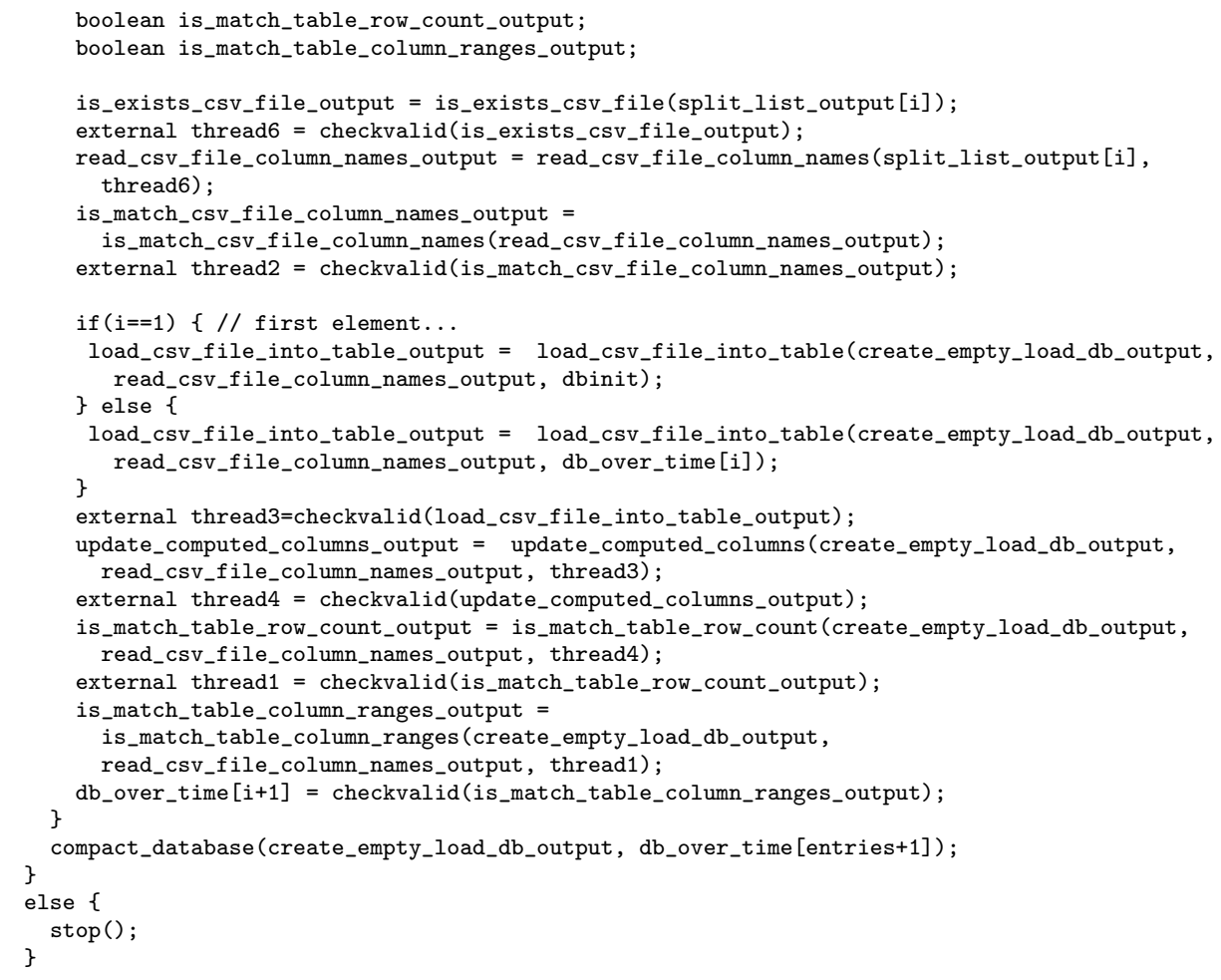

Core Query 1. The first query asks, for a given detection, which CSV files contributed to it. The strategy used to answer this query is to determine input CSV files that preceed, in the transitivity table, the process that inserted the detection. Suppose we want to determine the provenance of the detection that has the identifier 261887481030000003, the first query can be answered by first obtaining the Swift process identifier of the process that inserted the detection from the annotations included in the application database:

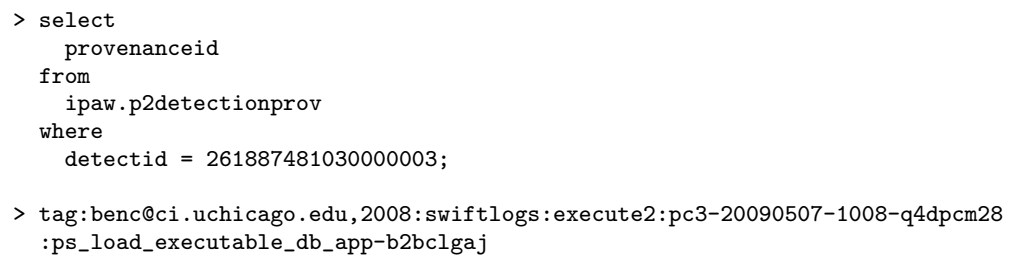

The identifier returned is an execute2 identifier, which means in this case that it refers to a successful execution attempt. In order to obtain the predecessors of this process in the transitivity table we need the actual execute identifier of the process, which can we can get with the following SQL query:

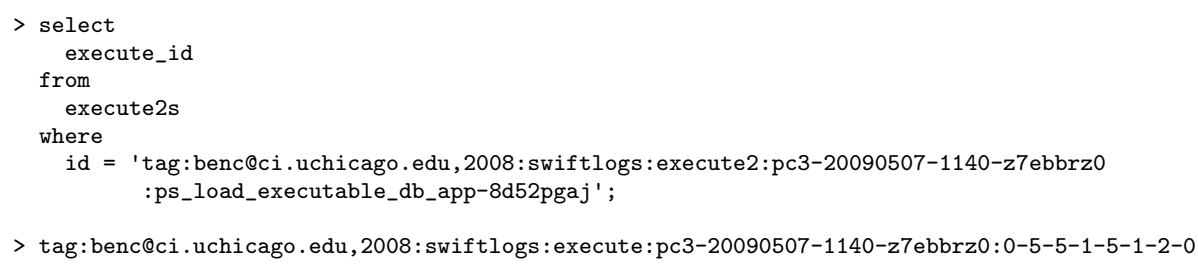

Finally, we determine the filenames of datasets that contain CSV inputs in the set of predecessors of the process that inserted the detection:

$>$ select

filename 


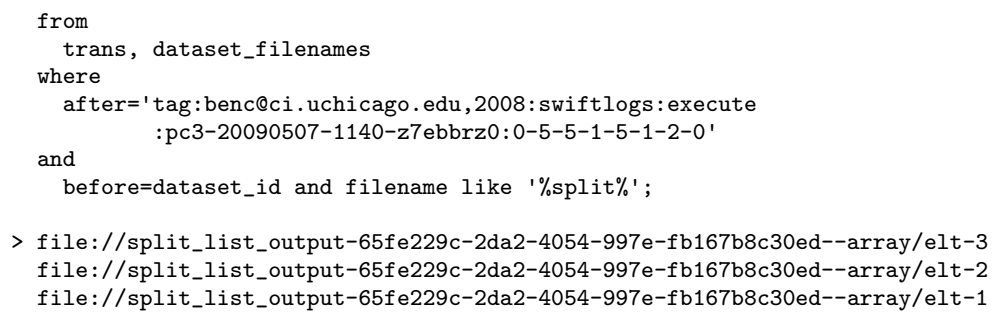

These files contain the filenames of the CSV files that were given as input to the workflow, and that will result in the detection row insertion:

P2_J062941_B001_P2fits0_20081115_P2Detection.csv, P2_J062941_B001_P2fits0_20081115_P2ImageMeta.csv, P2_J062941_B001_P2fits0_20081115_P2FrameMeta.csv

Core Query 2. The second query asks if the range check (IsMatchColumnRanges) was performed in a particular table, given that a user found values that were not expected in it. This is implemented in the q2. sh script in the Swift SVN repository with the following SQL query:

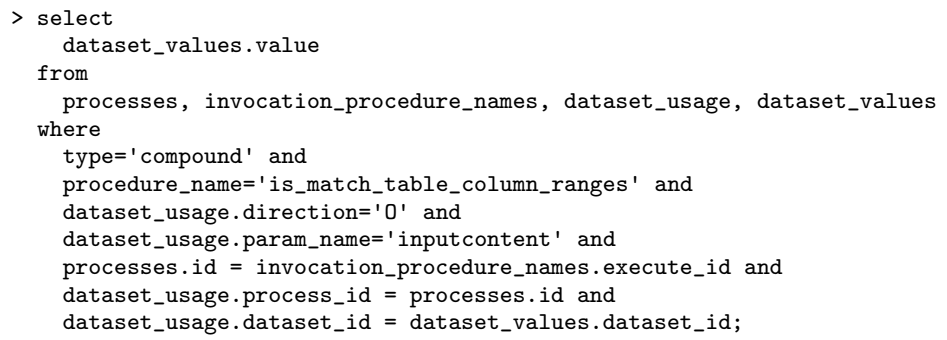

This returns the input parameter XML for all IsMatchColumnRanges calls. These are XML values, and it is necessary to examine the resulting XML to determine if it was invoked for the specific table. There is unpleasant cross-format joining necessary here to get an actual yes/no result properly, although probably could use a LIKE clause to peek inside the value.

Core Query 3. The third core query asks which operation executions were strictly necessary for the Image table to contain a particular (non-computed) value. This uses the additional annotations made, that only store which process originally inserted a row, not which processes have modified a row. So to some extent, rows are regarded a bit like artifacts (though not first order artifacts in the provenance database); and we can only answer questions about the provenance of rows, not the individual fields within those rows. That is sufficient for this query, though. First find the row that contains the interesting value and extract its IMAGEID. Then find the process that created the IMAGEID by querying the Derby database table P2IMAGEPROV:

$>$ select $*$ from ipaw.p2imageprov where imageid=6294301;

IMAGEID | PROVENANCEID

6294301 | tag:benc@ci.uchicago.edu,2008: swiftlogs: execute2:pc3-20090519

| -2057d8dyi9o9:ps_load_executable_db_app-dpc8q1bj

Now we have a process ID for the process that created the row. Now query the transitive closure table for all predecessors for that process (as in the first core query). This will produce all processes and artifacts that preceeded this row creation. Our answer differs from the sample answer because we have sequenced access to the database, rather than regarding each row as a proper first-order artifact. The entire database state at a particular time is a successor to all previous database accessing operations, so any process which led to any database access before the row in question is regarded as a necessary operations. This is undesirable in some respects, but desirable in others. For example, a row insert only works because previous database operations which inserted other rows did not insert a conflicting primary key - so there is data dependency between the different operations even though they operate on different rows. 
Optional Query 1. The workflow halts due to failing an IsMatchTableColumnRanges check. How many tables successfully loaded before the workflow halted due to a failed check? This counts how many load processes are known to the database (over all recorded workflows):

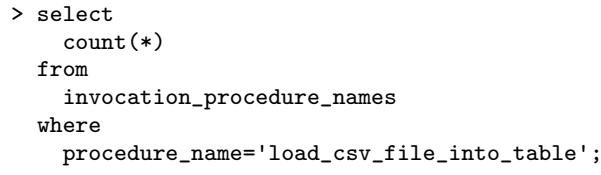

This can be restricted to a particular workflow run like this:

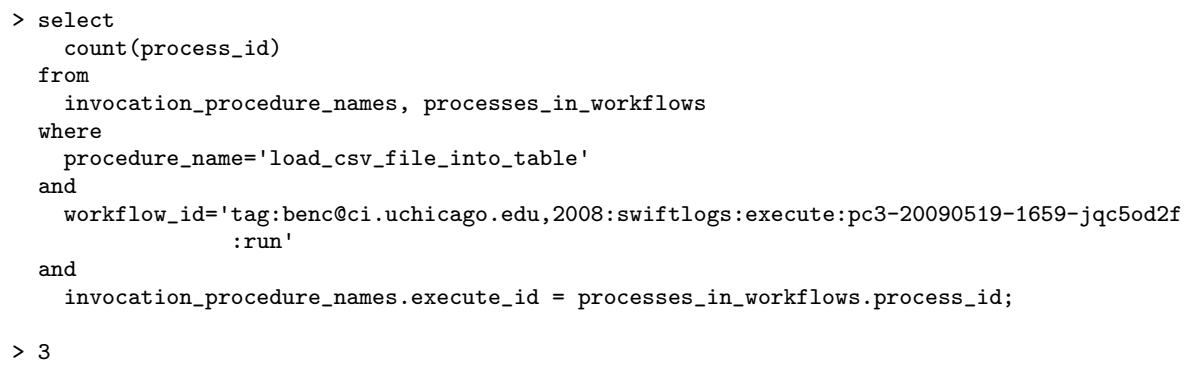

Optional Query 2. Which pairs of procedures in the workflow could be swapped and the same result still be obtained (given the particular data input)? In our Swift representation of the workflow, we control dataflow dependencies. So many of the activities that could be commuted are in our implementation run in parallel. One significant thing one cannot describe in SwiftScript (and so cannot answer from the provenance database using this method) is commuting operations on the database. From a Swift perspective, this is a limitation of our SwiftScript language rather than in the provenance implementation. The query lists which pairs Unix process executions (of which there are 50x50) have no data dependencies on each other. There are 2082 rows. The base SQL query is this:

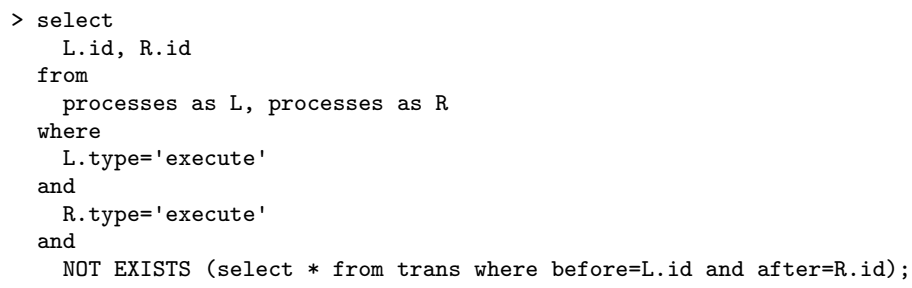

This answer is deficient in a few ways. We do not take into account non-execute procedures (such as compound procedures, function invocations, and operator executions) - there are 253 processes in total, 50 being executes and the remaineder being the other kinds of process. If we did that naively, we would not take into account compound procedures which contain other procedures (due to lack of decent support for nested processes - something like OPM accounts) and would come up with commutations which do not make sense.

In our initial attempts to implement LoadWorkflow, we found the use of the parallel foreach loop problematic because the database routines executed by the external application procedures are opaque to Swift. Due to dependencies between iterations of the loop, these routines were being incorrectly executed in parallel. It was necessary to serialize the loop execution to keep the database consistent. For the same reason, since most of the PC3 queries are for row-level database provenance, we had to implement a workaround for gathering this provenance by modifying the application database so that for every row inserted, an entry containing the execution identifier of the Swift process that performed this insertion is recorded on a separate annotation table.

The OPM output for a LoadWorkflow run in Swift was generated by a script that maps Swift's provenance data model to OPM's XML schema. Since OPM and Swift's provenance database use similar data models, 
Listing 3: Multiple provenance descriptions for a dataset.

int $\mathrm{a}=7$;

int $\mathrm{b}=10$

int $c[]=[a, b]$;

it is fairly straightforward to build a tool to import data from an OPM graph into the Swift provenance database. However we observed that the OPM outputs from the various participating teams, including Swift, carry many details of the LoadWorkflow implementation that are system specific, such as auxiliary tasks that are not necessarily related to the workflow. To answer the same queries, it would be necessary to perform some manual interpretation of the imported OPM graph in order to identify the relevant processes and artifacts.

\section{PC3: Evaluation}

PC3 provided an opportunity to use OPM in practice. This also enabled us to evaluate OPM and compare it to Swift's provenance data model. OPM originally did not specify a naming mechanism for globally identifying artifacts outside of an OPM graph. In Swift, dataset handles are given an URI, now OPM has an annotation for this purpose [18].

Swift's provenance implementation has two models of representing containment for dataset handles contained inside other dataset handles (arrays and complex types). A constructor/accessor model has special processes called accessors and constructors corresponding to the [] array accessor and $[1,2,3]$ explicit construction syntax in SwiftScript. This model is proposed in OPM. In the Swift implementation, this is a cause of multiple provenances for dataset handles. For example, consider the SwiftScript program displayed in listing 3, the expression c [0] evaluates to the dataset handle corresponding to the variable a. That dataset handle has a provenance trace indicating it was assigned from the constant value 7 . However, that dataset handle has additional provenance indicating that it was output by applying the array access operator [] to the array c and the numerical value 0 . In OPM, the artifact resulting from evaluating c [0] is distinct from the artifact resulting from evaluating a, although they may be annotated with an isIdenticalTo arc [15]. In order to address the divergence between OPM and Swift's provenance data model, the dataset handle implementation could be modified so that it supported dataset handles being aliases to other dataset handles. The alias dataset handle would behave identically to the dataset handle that it aliases, except that it would have different provenance reflecting both the provenance of the original dataset handle, and subsequent operations made to retrieve it. In listing 3, then, c [0] would return a newly created dataset handle that aliased the original dataset handle for a. There is also a container/contained model, where relations are stored directly between dataset handles indicating that one is contained inside the other, without intervening processes. These relations can be inferred from the constructor/accessor model. The Contained relation between two artifacts, defined in [15], indicates that one is contained within another. This maps relatively cleanly to Swift's in-memory model of dataset handles containing other dataset handles. Swift collections may be hierarchical. In [15], it is not specified if the Contained relation holds only one level deep or to all elements contained in a collection.

The Swift team [3] made a proposal [2] for a minor change to the XML schema to better reflect the perceived intentions of the OPM authors. It was apparent that the present representation of hierarchical processes in OPM is insufficiently rich for some groups and that it would be useful to represent hierarchy of individual processes and their containing processes more directly. In Swift this is given by two categories: at the highest level, SwiftScript language constructs, such as procedures and functions; below that, the mechanics of Swift's execution, such as moving files to and from computational resources, and interactions with job execution. Swift provenance work to date has concentrated on the high-level representation, treating all of the low-level behavior as opaque and exposing neither processes nor artifacts. An OPM modification proposal for this is forthcoming. In Swift, this information is often available through the Karajan [17] execution engine thread identifier which closely maps to the Swift process execution hierarchy: a Swift 
process contains another Swift process if its Karajan thread identifier is a prefix of the second processes Karajan thread identifier. The Swift provenance database stores values of dataset handles when those values exist in-memory (for example, when a dataset handle represents and integer or a string). During PC3, interest in a standard way to represent this was expressed.

\section{Related Work}

As pointed out by some provenance surveys [22] [8], provenance systems are diverse regarding what the subject of the recorded provenance information is, what its level of granularity is, and how it is gathered, stored, and queried. This is also noticeable in PC3, where a variety of approaches were used to perform its activities. These provenance systems use a variety of data models, including semantic, relational and semistructured ones. Nevertheless, most of them were able to map their data models to OPM. In this section we build on these surveys and PC3 to compare Swift to other provenance systems.

Some provenance systems are not dependent on a particular workflow management system and work as provenance stores accessible, for example, as web services or through API calls. Karma [4], for instance, is implemented as a web service and stores provenance information in a relational database. Another example is Tupelo [20], which is a data and metadata management middleware that uses semantic web techniques, it has an API for enabling the storage of provenance information. Unlike Swift, systems of this type often require the instrumentation of the applications that compose a scientific workflow, which was also observed during PC3. This may prove difficult when the users of these applications are not also their developers, or if these applications are given by undocumented legacy code.

Another category is given by workflow systems that have integrated provenance management support. Vistrails [14], for instance, has a specialized provenance query language and uses both XML and relational databases to store provenance about data, processes and workflow evolution. This category includes Swift, which has a provenance system that is tightly coupled to it, that gathers information both about processes and data involved in the execution of a parallel script. It has comprehensive support for execution engines on high-performance parallel and distributed computing environments. Also, by having an integrated provenance system, Swift enables its users to readily generate and query provenance records of their experiments. In Swift, prospective provenance [26] is given by SwiftScript code, workflow evolution is not recorded. One alternative for recording this, not yet implemented, would be to couple Swift's provenance database with a source code version control system.

\section{Concluding Remarks}

Swift was able to perform the activities proposed for PC3. This success illustrates its capability to support provenance collection and analysis. Its provenance model is close to OPM, which enables interoperability with other provenance systems. One important aspect of Swift is its support for scalable execution of large-scale computations on parallel and distributed environments, along with the collection of provenance information. Several examples of parallel scripting applications are mentioned in [23], which include protein structure prediction, identification of drug targets using computational docking, and computational economics. In a recent example, Swift executed a neural imaging analysis workflow that involved 500,000 jobs. Swift development continues with the objective of improving its provenance capabilities and future work will concentrate on the following aspects:

Provenance system scalability. Swift provenance tracking model results in the generation and storage of large amounts of data. For smaller computations, such as LoadWorkflow, this is not a problem, but for larger computations (which is precisely the sort of computations for which Swift is particularly well suited) the provenance can become extremely large, and provenance queries can take a long time to execute. It may be desirable to provide options that can allow the programmer to request that Swift store less data albeit (presumably) with reduced accuracy. It may also be interesting to use distributed data management techniques to enable better scalability.

Provenance query system. It was clear from PC3 that although it is possible to express the provenance queries in SQL it is not always straightforward to do so, due to its poor transitivity support. One future 
objective is to make the provenance query system, which should include a specialized provenance query language, capable of being readily queried by scientists to let them do better science through validation, collaboration, and discovery.

\section{Acknowledgments}

This work was supported in part by CAPES Grant 5205-09-3, CNPq, and the Office of Advanced Scientific Computing Research, Office of Science, U.S. Department of Energy, under Contract DE-AC02-06CH11357.

\section{A. Database Schema}

This is the schema definition used for the main relational provenance implementation (in both SQLite3 and PostgreSQL):

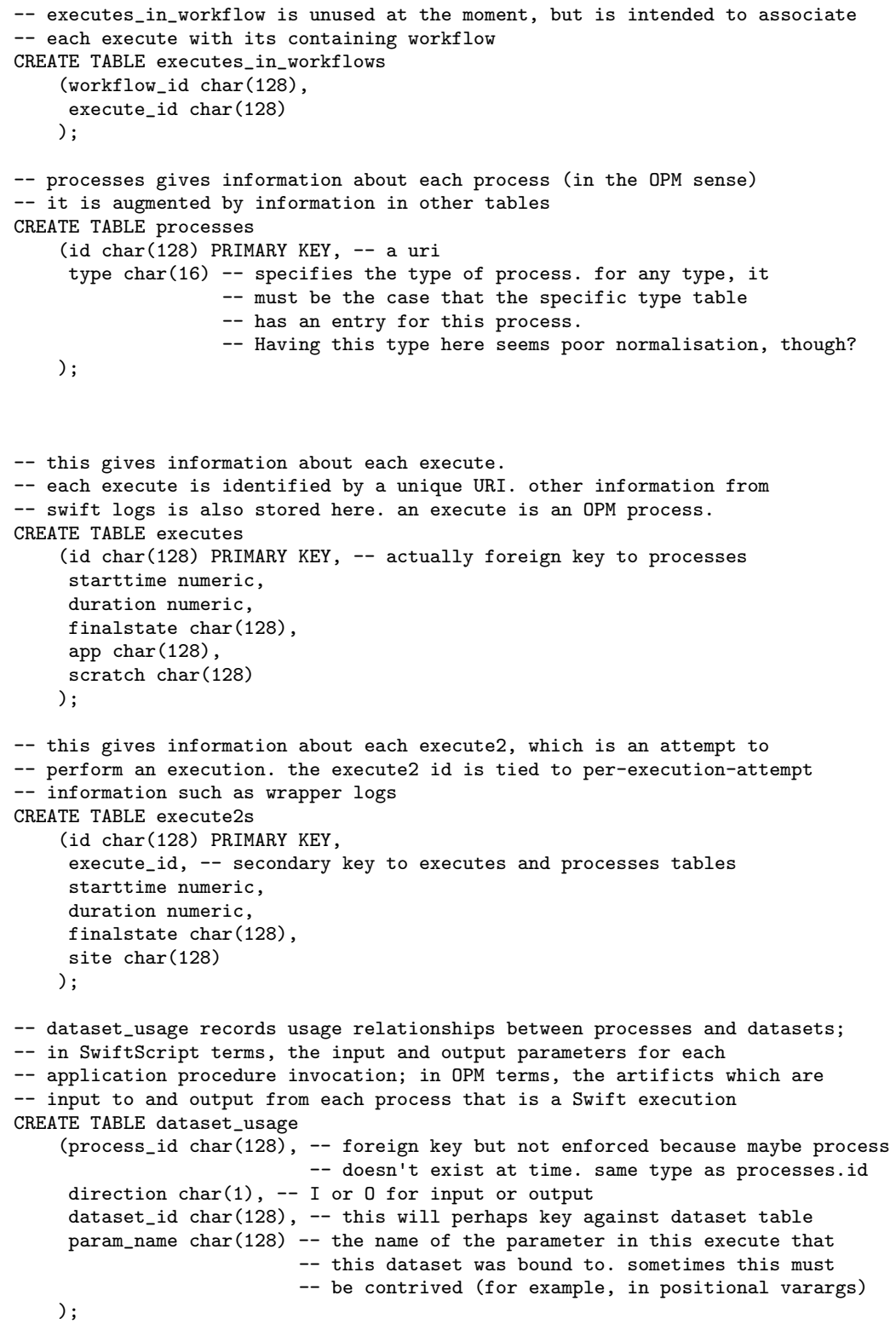


[4] B. Cao, B. Plale, G. Subramanian, E. Robertson, and Y. Simmhan. Provenance Information Model of Karma Version 3. In Proc. IEEE Congress on Services, pages 348-351, 2009.

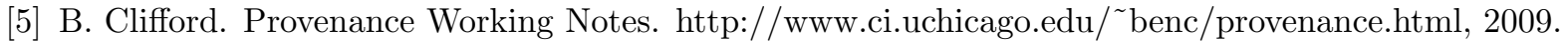

[6] B. Clifford, I. Foster, J. Voeckler, M. Wilde, and Y. Zhao. Tracking provenance in a virtual data grid. Concurrency and Computation: Practice and Experience, 20(5):565-575, 2008.

[7] K. Czajkowski, I. Foster, N. Karonis, C. Kesselman, S. Martin, W. Smith, and S. Tuecke. A Resource Management Architecture for Metacomputing Systems. In Job Scheduling Strategies for Parallel Processing - IPPS/SPDP '98 Workshop, volume 1459 of LNCS, pages 62-82. Springer, 1998.

[8] S. da Cruz, M. Campos, and M. Mattoso. Towards a Taxonomy of Provenance in Scientific Workflow Management Systems. In Proc. IEEE Congress on Services, Part I, (SERVICES I 2009), pages 259-266, 2009.

[9] E. Deelman, D. Gannon, M. Shields, and I. Taylor. Workflows in e-Science: An overview of workflow system features and capabilities. Future Generation Computer Systems, 25(5):528-540, 2009.

[10] G. Dong, L. Libkin, J. Su, and L. Wong. Maintaining Transitive Closure of Graphs in SQL. Intl. Journal of Information Technology, 5, 1999.

[11] D. Epemaa, M. Livny, R. van Dantzig, X. Evers, and J. Pruyne. A worldwide flock of Condors: Load sharing among workstation clusters. Future Generation Computer Systems, 12(1):53-65, 1996.

[12] N. Kaiser et al. Pan-STARRS: A Large Synoptic Survey Telescope Array. Proc. SPIE, 4836:154-164, 2002.

[13] I. Foster, J. Vockler, M. Wilde, and Y. Zhao. Chimera: A Virtual Data System for Representing, Querying and Automating Data Derivation. In Proc. 14th International Conference on Scientific and Statistical Database Management (SSDBM'02), pages 37-46, 2002.

[14] J. Freire, C. Silva, S. Callahan, E. Santos, C. Scheidegger, and H. Vo. Managing Rapidly-Evolving Scientific Workflows. In International Provenance and Annotation Workshop (IPAW 2006), volume 4145 of $L N C S$, pages 10-18, 2006.

[15] P. Groth, S. Miles, P. Missier, and L. Moreau. A Proposal for Handling Collections in the Open Provenance Model. http://mailman.ecs.soton.ac.uk/pipermail/provenance-challenge-ipaw-info/2009June/000120.html, 2009.

[16] R. Henderson. Job Scheduling Under the Portable Batch System. In Job Scheduling Strategies for Parallel Processing - IPPS '95 Workshop, volume 949 of LNCS, pages 279-294. Springer, 1995.

[17] G. Laszewski, M. Hategan, and D. Kodeboyina. Java CoG Kit Workflow. In I. Taylor, E. Deelman, D. Gannon, and M. Shields, editors, Workflows for e-Science, pages 340-356. Springer, 2007.

[18] L. Moreau, B. Clifford, J. Freire, Y. Gil, P. Groth, J. Futrelle, N. Kwasnikowska, S. Miles, P. Missier, J. Myers, Y. Simmhan, E. Stephan, and J. Van den Bussche. The Open Provenance Model - Core Specification (v1.1). Future Generation Computer Systems, doi:10.1016/j.future.2010.07.005, 2010.

[19] L. Moreau, Y. Zhao, I. Foster, J. Voeckler, and M. Wilde. XDTM: XML Dataset Typing and Mapping for Specifying Datasets. European Grid Conference (EGC 2005), 2005.

[20] J. Myers, J. Futrelle, J. Plutchak, P. Bajcsy, J. Kastner, L. Marini, R. Kooper, R. McGrath, T. McLaren, A. Rodríguez, and Y. Liu. Embedding Data within Knowledge Spaces. CoRR, abs/0902.0744, 2009.

[21] I. Raicu, Y. Zhao, C. Dumitrescu, I. Foster, and M. Wilde. Falkon: A Fast and Lightweight Task Execution Framework. In Proc. ACM/IEEE Conference on High Performance Networking and Computing (Supercomputing 2007), 2007. 
[22] Y. Simmhan, B. Plale, and D. Gannon. A Survey of Data Provenance in e-Science. SIGMOD Record, 34(3):31-36, 2005.

[23] M. Wilde, I. Foster, K. Iskra, P. Beckman, A. Espinosa, M. Hategan, B. Clifford, and I. Raicu. Parallel Scripting for Applications at the Petascale and Beyond. IEEE Computer, 42(11):50-60, November 2009.

[24] T. Ylönen. SSH - Secure Login Connections over the Internet. In Proc. of the Sixth USENIX Security Symposium, pages 37-42, 1996.

[25] Y. Zhao, M. Hategan, B. Clifford, I. Foster, G. Laszewski, I. Raicu, T. Stef-Praun, and M. Wilde. Swift: Fast, Reliable, Loosely Coupled Parallel Computation. In Proc. 1st IEEE International Workshop on Scientific Workflows (SWF 2007), pages 199-206, 2007.

[26] Y. Zhao, M. Wilde, and I. Foster. Applying the Virtual Data Provenance Model. In International Provenance and Annotation Workshop (IPAW 2006), volume 4145 of LNCS, pages 148-161. Springer, 2006. 


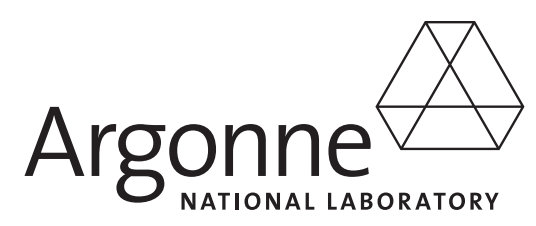

\section{Mathematics and Computer Science Division}

Argonne National Laboratory

9700 South Cass Avenue, BIdg. 240

Argonne, IL 60439-4847

www.anl.gov 